\title{
Double-Stranded RNA Dependent Protein Kinase (PKR) Deficiency Protects the Liver Against Ischemia/reperfusion Injury
}

\section{Da Tang}

Central South University Third Xiangya Hospital https://orcid.org/0000-0001-7178-0962

\section{Youxiang Jiang}

Central South University Third Xiangya Hospital

\section{Yiting Tang}

Central South University

\section{Xianhui Qiu}

Central South University Third Xiangya Hospital

\section{Xiaoye Cheng}

Central South University

\section{Wenbo Li}

Central South University

\section{Wenwu Cai}

Central South University

\section{Daming Li}

Central South University

Timothy R Billiar ( $\nabla$ billiartr@upmc.edu )

University of Pittsburgh https://orcid.org/0000-0002-2949-0811

Ben Lu

Central South University

\section{Research Article}

Keywords: PKR, Hepatic ischemia/reperfusion injury, STAT1, HMGB1, Inflammation, Cell death

Posted Date: November 23rd, 2021

DOI: https://doi.org/10.21203/rs.3.rs-1077567/v1

License: (c) (1) This work is licensed under a Creative Commons Attribution 4.0 International License.

Read Full License 


\section{Abstract}

Background: Interruption of hepatic blood inflow to reduce intraoperative blood loss during liver surgery such as elective liver surgical procedures and organ transplantation causes hepatic ischemia and subsequent reperfusion that leads to liver damage and dysfunction. Double-stranded RNA dependent protein kinase (PKR) is an important regulator of immune responses in the settings of infection and tissue injury. However, little is known about the function of PKR in liver ischemia/reperfusion (I/R) injury.

Methods: Wild-type (WT) and PKR knockout $\left(\mathrm{PKR}^{-/-}\right)$mice were subjected to partial (70\%) hepatic I/R injury. In another set of experiments, mice were pretreated intraperitoneally with $\mathrm{C} 16$, a specific PKR inhibitor, or fludarabine, a specific inhibitor of STAT1 (signal transducer and activator of transcription 1), then subjected to I/R in-vivo. In-vitro investigations were conducted by subjecting primary mouse hepatocytes with hypoxia. The role of PKR in I/R-induced liver injury and the potential underlying molecular mechanisms were clarified through biological and phenotypic analyses.

Results: Liver PKR protein levels were increased during the ischemic and reperfusion stages in both the mouse liver I/R model and the livers of humans who were subjected to liver resection. PKR inactivation by genetic deletion or pharmacological inhibition exhibited less liver damage than their respective control. PKR deficiency was also associated with lower levels of inflammatory cytokines and less apoptotic cell death. Mechanistically, STAT1 signaling was inhibited in livers of $\mathrm{PKR}^{-/-}$mice but was activated in WT mice. Most importantly, WT mice treated with STAT1 specific inhibitor fludarabine were protected from hepatic I/R injury. PKR also regulated the release of HMGB1 in hepatic I/R injury.

Conclusions: Our novel findings document PKR is a modulator in I/R-induced liver damage. Inhibition of PKR by using the specific inhibitor, C16, may represent a promising therapeutic target for the management of $\mathrm{I} / \mathrm{R}$ injury-related diseases.

\section{Introduction}

Hepatic ischemia/reperfusion (I/R) injury is an unavoidable consequence of many clinical settings, particularly during liver transplantation, liver resection, trauma and hemorrhagic shock, and can result in liver dysfunction and failure (Zhang et al. 2021, Zhang et al. 2018). During the ischemic period, the interruption of oxygen leads to hepatocyte damage. Subsequently, the restoration of blood flow triggers innate immune responses and exaggerated local inflammation (Sun et al. 2015). In addition, reactive oxygen species (ROS)-mediated hepatocellular necrosis and inflammatory response-induced apoptosis can also contribute to liver I/R injury (Liu et al. 2019, Yazdani et al. 2021). Despite the progress in understanding the mechanisms behind I/R-induced liver injury, no effective preventative treatments have advanced into clinical use (Jaeschke et al. 2012). Thus, effective pharmacological treatments for individuals subjected to I/R-induced liver injury are urgently needed. In order to achieve this, a deeper understanding of the molecular and cellular events underlying this pathogenic process may be required. 
Double-stranded RNA dependent protein kinase (PKR) is a ubiquitously expressed protein kinase that was initially recognized as an interferon-inducible antiviral protein (Meurs et al. 1990). When PKR is activated by double-stranded (ds) RNA, it undergoes homodimerization and then promotes the phosphorylation of elF2a, thus leading to inhibition of protein synthesis (Holcik et al. 2005). In addition to its established key role in antiviral activities, previous studies have demonstrated that PKR is also involved in a series of pathophysiological events including inflammation (Hsu et al. 2004), cancers (Yoon et al. 2009, Kim et al. 2002), neurodegeneration (Hugon et al. 2017) and metabolic diseases (Nakamura et al. 2010). We have previously shown that PKR is also required for the activation of the NLRP3 inflammasome (Lu et al. 2012), however, whether PKR is implicated in hepatic I/R injury has not been previously described.

In the current study, we aimed to determine the role of PKR in the pathogenesis of hepatic l/R injury. Using both $\mathrm{PKR}^{-/-}$mice and WT mice treated with a specific PKR inhibitor, we find significant roles for PKR in $\mathrm{I} / \mathrm{R}$-induced liver injury and inflammation. Our results suggest that PKR and its downstream signaling intermediates could be interesting targets to prevent I/R injury in the liver.

\section{Materials And Methods}

\section{Reagents}

Western blot antibodies: GAPDH (\# ab181602), PKR (\#ab184257) and HMGB1 (\# ab79823) antibodies were purchased from Abcam. STAT1 (\# 14994T) and $\beta$-actin antibodies were obtained from Cell Signaling Technologies. ELISA kits specific for IL-6 and IL-1 $\beta$ were purchased from Invitrogen. C16 (\#527450) was obtained from Sigma-Aldrich. Fludarabine (\#S1491) was purchased from Selleck Chemicals. The terminal deoxynucleotidyl transferase-mediated dUTP nick-end labeling kit (KGA7072) was purchased from KeyGEN BioTECK (Jiangsu, China).

\section{Animals and treatment}

Animal protocols were approved by the Ethics Committee of Central South University. Male wild-type (C57BL/6J) mice were purchased from Hunan SJA Laboratory Animals Co. Ltd and PKR-defective mice were bred at our facility. In experiments to inhibit the activation of PKR, the PKR inhibitor C16 or an equal volume vehicle was injected intraperitoneally at $500 \mathrm{ug} / \mathrm{kg}$ QD for three days prior to the ischemic insult. To further block the STAT1, fludarabine, a specific inhibitor of STAT1, was administered i.p at $40 \mathrm{mg} / \mathrm{kg}$ QD for 7 days before the treatment of I/R injury (Pan et al. 2013). All animals that were used in the present study were maintained in a specific pathogen-free atmosphere with a 12-h light/dark cycle. Only 8-12-wk-old mice were used at a mass of $25-28 \mathrm{~g}$.

\section{Mouse liver ischemia/reperfusion (I/R) injury model}

A nonlethal established model of segmental (70\%) hepatic I/R was used as described previously (Tsung et al. 2005). Briefly, after the mice were anesthetized, arterial/portal venous blood supply to the left and median liver lobes was interrupted by using an atraumatic microvascular clamp under aseptic conditions 
for 60 minutes, thereby initiating hepatic reperfusion. The ambient temperature of the mice was maintained at $33^{\circ} \mathrm{C}$ using a warming incubator chamber during the ischemia phase. Sham-operated mice were subjected to the same surgical procedure without hepatic ischemia. Animals were euthanized at $6 \mathrm{~h}$ after reperfusion, and serum and liver samples were collected.

\section{Serum sample assays}

Serum alanine aminotransferase (ALT) and serum aspartate aminotransferase (AST) levels were measured using a Chemistry System autoanalyzer. Serum levels of IL- 6 and IL-1 $\beta$ were analyzed with ELISA kits according to the manufacturer's instructions (Invitrogen). HMGB1 was also quantified by using ELISA (IBL International).

\section{H\&E staining and scoring method}

Liver sections were stained with hematoxylin and eosin (H\&E) as described (Li et al. 2019). Image J software was used to analyze necrotic areas. All slides were blindly quantified in more than 3 high-power fields ( $\times 10$ magnification) to evaluate the necrotic area in each section. Samples were scored by three or four independent members of each group according to Suzuki's score, based on a scale of 0-4, for assessing liver damage I/R (Suzuki et al. 1993).

\section{TUNEL assay}

Cell death in tissue paraffin sections was analyzed by the TUNEL staining method following the manufacturer's protocol. Images were obtained by confocal microscopy. The numbers of dead cells in the liver sections were blindly assessed by counting the labeled cells in high-power fields (HPFs)/sections under confocal microscopy (40x).

\section{Primary hepatocyte isolation and treatment.}

Mice were perfused by an in situ collagenase (type VI, Sigma) technique as previously described (Yi et al. 2020). Hepatocytes were separated from nonparenchymal cells (NPCs) by low-speed centrifugation. Hepatocyte purity exceeded $98 \%$ as evaluated by light microscopy. Trypan blue exclusion was performed

to determine the cell viability of the hepatocytes (>95\%). Hepatocytes were plated on gelatin-coated culture plates in Williams' E medium with 10\% calf serum, $1 \mu \mathrm{M}$ insulin, $15 \mathrm{mM}$ HEPES, $2 \mathrm{mM} \mathrm{L-}$ glutamine, $100 \mathrm{U} / \mathrm{mL}$ streptomycin, and $100 \mathrm{U} / \mathrm{mL}$ penicillin. Cells were allowed to attach to plates for at least $4 \mathrm{~h}$ before treatment. For experiments involving hypoxia, the culture medium was replaced with fresh medium, and hepatocytes were placed into a modular incubator chamber, which was equilibrated with the anoxic gas mixture $\left(5 \% \mathrm{CO}_{2}, 94 \% \mathrm{~N}_{2}\right.$, and $\left.1 \% \mathrm{O}_{2}\right)$.

\section{Human samples}

The human liver tissues used in this study were obtained from patients who were subjected to elective hepatectomy due to hepatocellular carcinoma. Liver samples were acquired from tumor-free liver tissue 
from the individuals at two time points: before ischemia (baseline) and after ischemia but before reperfusion (ischemia period). Informed consent forms were signed by all patients. These procedures were approved by The Second Xiangya Hospital of Central South University Review Board.

\section{Immunofluorescence}

The WT and $\mathrm{PKR}^{-/}$primary hepatocytes were seeded on glass slides placed into 6-well plates at a density of $2 \times 10^{5}$ cells $/ \mathrm{mL}$ and subjected to normoxia or hypoxia for indicated time points. Then cells were washed with PBS three times and fixed with $4 \%$ paraformaldehyde for $15 \mathrm{~min}$. Fixed hepatocytes were permeabilized with $0.1 \%$ Triton X-100 for 15 min, blocked with $2 \%$ BSA for 2 hours, and incubated with anti-HMGB1 antibodies (1:250) (Abcam, 79823) overnight. After washing with PBS, fixed cells were treated with conjugated anti-rabbit secondary antibody (1:500) and F-actin (1:100) for 60 min. Nuclei were counterstained with DAPI for 5-10 min. Cells in each group were imaged using an inverted fluorescence microscope (Carl Zeiss Microscopy GmbH, Germany).

\section{Lactate dehydrogenase (LDH) assay.}

Culture media were collected and centrifuged to sediment cells. Lytic cell death following normoxia or hypoxia treatment was analyzed by measurement of LDH released into the cell medium. At the end of treatment, the cell supernatant was subjected to the LDH Cytotoxicity Assay (Beyotime Biotechnology, China).

\section{Western blot}

Western blotting analysis was performed using whole lysates from liver tissue or proteins from cell-free supernatants that were extracted by methanol-chloroform precipitation. Briefly, samples were subjected to electrophoretic separation on 8-12\% SDS-PAGE gels and transferred onto PVDF membranes. PBST (with $5 \%$ skim milk powder) was used to block nonspecific binding sites for $1 \mathrm{~h}$ at room temperature. After that, the membranes were incubated with primary antibodies at $4{ }^{\circ} \mathrm{C}$ overnight. Membranes were washed three times with PBST for 10 minutes, incubated with secondary antibody for 1 hour, and then washed another three times for 10 minutes in PBST before being detected for chemiluminescence (Bio-Rad, USA). Protein expression levels were quantified with ImageJ software and normalized to GAPDH or $\beta$-actin as a loading control.

Statistical analysis: All quantitative data were analyzed using GraphPad Prism and presented as mean \pm SEM. Differences between the two experimental groups were evaluated by Student's $t$ test. $P<0.05$ was considered statistically significant.

\section{Results}


To clarify whether PKR is involved in hepatic I/R injury, we first evaluated PKR expression in WT mice subjected to partial (70\%) hepatic ischemia followed by reperfusion. Western blotting revealed detectable PKR expression at baseline and this was increased almost 4-fold at $6 \mathrm{~h}$ after liver I/R (Fig.1A, B). To further address the human relevance of these findings, we compared PKR expression in liver tissues from patients with hepatic I/R during elective liver resection. Liver samples from the same individual were obtained at baseline (before ischemia) and after ischemia resulting from inflow occlusion during the procedure. In line with the increased PKR protein levels in the mouse model, PKR expression in human subjects who underwent hepatectomy was also higher during the ischemic phase than at baseline (Fig.1C, D). The induced PKR expression in this process demonstrates the possibility of the involvement of PKR during hepatic I/R injury.

\section{PKR exacerbates hepatic l/R injury}

We assessed the role of PKR in I/R-induced liver damage using global PKR knockout mice. PKR deficiency was first confirmed in the livers of $\mathrm{PKR}^{-/-}$mice (Fig.2A). Subsequently, WT and $\mathrm{PKR}^{-/-}$mice were treated with warm hepatic I/R with 1 hour of ischemia followed by $6 \mathrm{~h}$ of reperfusion. Liver damage was evaluated by morphological and biochemical indices. Serum ALT and AST levels were notably lower in $\mathrm{PKR}^{-/-}$mice compared with those of WT mice subjected to I/R injury (Fig.2B, C). Additionally, histological analysis showed significantly less liver necrosis in tissue sections from $\mathrm{PKR}^{-/-}$mice after $6 \mathrm{~h}$ of reperfusion (Fig.2D, E), and this was associated with lower Suzuki's (on a scale from 0-4) liver injury scores (Fig.2F). To further explore the role of PKR in liver I/R injury, C16, a specific inhibitor of PKR was administrated intraperitoneally before the ischemic insult. Pharmacological inhibition of PKR also significantly reduced circulating levels of ALT and AST (Fig.2G, H) and led to smaller areas of necrosis than those seen in the vehicle-treated I/R group (Fig.2I-K). Taken together, these findings indicate PKR inactivation by genetic deletion or pharmacological inhibition confers protection against hepatic l/R injury.

\section{PKR participates in I/R-induced inflammatory responses}

Liver I/R injury involves both direct cellular damage induced by oxidative stress and injury due to the activation of inflammatory responses. Our previous study showed that PKR deficiency severely impairs inflammasome activation, thereby inhibiting the release of IL-1 $\beta$ and IL-18 in E.coli-induced sepsis (Lu et al. 2012). To determine whether PKR deletion could ameliorate hepatic I/R injury by regulating inflammatory responses, we examined circulating levels of representative inflammatory cytokines. We found that the PKR deletion resulted in lower systemic inflammation after hepatic l/R, as demonstrated by lower serum levels of IL-6 in $\mathrm{PKR}^{-/-}$mice compared to control WT mice (Fig 3A). However, levels of IL-1 $\beta$ after I/R were not influenced by the ablation of PKR (Fig.3B). Mitogen-activated protein kinase (MAPK) regulates both the inflammatory response and cell survival during I/R injury (Nace et al. 2013). Protein levels of the phosphorylated forms of three MAPK proteins, including extracellular signal-regulated kinase (ERK), P38 and c-Jun-N-terminal kinase (JNK), were analyzed in the livers of mice subjected to I/R insult (Fig.3C). Levels of p-ERK and p-JNK were higher after I/R compared to Sham 
controls at $6 \mathrm{~h}$ (Fig.3C-F). However, protein levels of p-ERK and p-JNK were lower after I/R in PKR ${ }^{-/-}$mice compared with WT I/R groups (Fig.3C-F). These results show that PKR deficiency inhibits systemic inflammation in response to liver I/R injury, and PKR modulates I/R-induced activation of ERK and JNK signaling.

\section{PKR is associated with apoptotic cell death after hepatic I/R injury}

I/R injury is associated with both cellular necrosis and apoptosis (Brenner et al. 2013). To determine whether I/R-induced cell death was associated with PKR activity, TUNEL staining was performed on liver sections from the ischemic lobe from WT and $\mathrm{PKR}^{-/-}$mice. I/R resulted in a marked increase in TUNELpositive cells in WT mice and this was significantly reduced in liver sections of $\mathrm{PKR}^{-/-}$mice after $6 \mathrm{~h}$ of reperfusion (Fig.4A, B). During I/R-induced cell damage, pro-apoptotic/pro-necrotic factors and negative regulators of cell death were measured by western blotting (Fig.4C). Protein expression levels of cleavedcaspase 3 were reduced in the $\mathrm{PKR}^{-/-} \mathrm{I} / \mathrm{R}$ group but were higher in liver samples from WT mice (Fig. $4 \mathrm{C}, \mathrm{F}$ ). Unexpectedly, Western blotting showed that the levels of Bax and Bcl-2 were comparable between WT and $\mathrm{PKR}^{-/}$mice (Fig.4C-E). These data reveal that PKR expression is associated with more apoptotic cell death after liver $\mathrm{I} / \mathrm{R}$, however, this does not appear to involve regulation of $\mathrm{Bax}$ or $\mathrm{Bcl} 2$.

\section{PKR regulates hepatic I/R insult in a STAT1-dependent manner}

The STAT (signal transducer and activator of transcription) family of proteins exerts a crucial role in many cellular processes, including cell growth and proliferation as well as apoptotic cell death. Previous studies also revealed that PKR could negatively or positively regulate STAT1 in different contexts (Hsu et al. 2004, Wang et al. 2006). Thus, we next examined the potential role of STAT1 signaling on PKRinduced aggravation of hepatic I/R injury. STAT1 signaling was activated in I/R-induced liver injury (Fig.5A). Additionally, we found that phosphorylated protein levels of STAT1 in the PKR ${ }^{-/-} \mathrm{I} / \mathrm{R}$ group were significantly lower compared with livers from the WT I/R group (Fig.5A). However, total STAT1 protein levels were unchanged between these groups (Fig.5A). To further elucidate whether the STAT1 signaling contributes to PKR-mediated liver injury in vivo, STAT1 was blocked by its specific inhibitor, fludarabine. Western blotting showed administration of fludarabine before liver I/R suppressed the expression of STAT1 (Fig. 5B). Histological assessment of the ischemic lobe and evaluation of serum ALT and AST revealed that fludarabine treatment markedly ameliorated liver damage in WT mice compared with vehicle-treated I/R group (Fig.5C-G). These in vivo findings indicate that PKR-mediated exacerbation following liver I/R injury involves the STAT1 pathway.

\section{HMGB1 release is dependent on PKR in I/R induced liver injury}

HMGB1 is a nuclear protein that has been shown to contribute to liver I/R injury when released from ischemic and dying hepatocytes (Tsung et al. 2005), which revealed that released HMGB1 after hepatic I/R functioned as a DAMP (damage-associated molecular pattern). We had also demonstrated that PKR deletion or pharmacological inhibition of JAK/STAT1 signaling prevents LPS-induced HMGB1 release (Lu 
et al. 2012, Lu et al. 2014). Therefore, we sought to examine the role of PKR or STAT1 in the release of HMGB1 during hepatic I/R injury. We found that $\mathrm{PKR}^{-/-} \mathrm{I} / \mathrm{R}$ mice had significantly lower serum HMGB1 levels compared to control WT I/R group (Fig.6A). Consistent with the results of gene knockout mice, serum HMGB1 levels were notably lower in WT mice treated with the PKR inhibitor C16 compared to the vehicle-treated I/R group (Fig.6B). During the process of I/R injury, pharmacological inhibition of STAT1 also suppressed increases in circulating HMGB1 levels in comparison with the vehicle-treated group (Fig.6C). Hypoxia is considered as an initiating event in the I/R insult. To explore whether PKR participates in regulating the release of HMGB1 in hypoxic hepatocytes, the cellular localization of HMGB1 using immunofluorescence staining was performed in cultured hepatocytes that underwent normoxia or hypoxia (1\% oxygen) for 20h. HMGB1 translocation to the cytoplasm was observed in WT hepatocytes, which was not observed in $\mathrm{PKR}^{-/-}$hepatocytes (Fig.6D). HMGB1 protein levels in the media of WT hepatocytes were also higher than that measured in the supernatants from $\mathrm{PKR}^{-/}$cells (Fig.6E) subjected to hypoxia. However, there was no difference in cell death between WT and PKR ${ }^{-/-}$cells that were exposed to hypoxia for $20 \mathrm{~h}$ (Fig.6F). These data demonstrate that PKR is essential for the HMGB1 nucleocytoplasmic transfer and release after $\mathrm{I} / \mathrm{R}$ in vivo and hypoxic stress in vitro.

\section{Discussion}

PKR has been identified as a danger-sensing molecule that contributes to multiple pathophysiological processes. In addition to IFN and dsRNA, PKR can be upregulated by PAMPs (pathogen-associated molecular pattern molecules) and DAMPs, such as TNF-a (Yeung et al. 1996), IL-1 (Horng et al. 2001), LPS (Lu et al. 2012), and heat shock proteins (Donzé et al. 2001). In the present study, we discovered that PKR protein levels were upregulated in mice after hepatic I/R and in human liver tissues due to ischemia, which suggests that PKR also serves as a stress sensor during tissue I/R.

C16 is a small molecule that can suppress the activation of PKR and prevents the translational block that follows PKR exposure (Jammi et al. 2003, Shimazawa et al. 2006). C16 has been extensively used to determine the role of PKR both in vivo (Xiao et al. 2016) and in vitro (Watanabe et al. 2020). The dose of C16 in this study was based on previous experience in which mice received C16 i.p. at $500 \mathrm{ug} / \mathrm{kg}$ (Couturier et al. 2012, Li et al. 2017). Thus, C16 might be a promising pharmacological treatment that could be translated into clinical application for hepatic I/R injury.

Liver I/R insult combines ischemia-triggered cell death and inflammation-induced reperfusion injury (Brenner et al. 2013). Initial parenchymal cell damage releases DAMP that promotes the production of cytokines and chemokines. This inflammatory response, in turn, aggravates hepatocellular injury (van Golen et al. 2013). Previous studies showed that increased levels of circulating TNF-a and IL-6 were associated with infections after human liver transplantation (Hamilton et al. 1993). We also revealed that PKR deletion inhibited E.coli-induced inflammatory responses in sepsis (Lu et al. 2012). Consistent with these findings, we demonstrate here that PKR knockout reduced the circulating levels of inflammatory cytokines (IL-6) following hepatic I/R injury. These in vivo experiments implicate PKR in the inflammatory responses that follow hepatic I/R injury. Two types of cell death, apoptosis and necrosis, occur after 
hepatic I/R, especially during the reperfusion stage. It has been reported that PKR is involved in macrophage apoptosis in response to bacteria (Hsu et al. 2004). Another study also suggested that PKR was a key factor in inducing apoptosis in response to oxidative stress (Li et al. 2010). Here, we found that PKR contributed to apoptotic cell death after hepatic I/R injury. Our results suggest that this may result from inflammatory signaling activated by PKR.

Several lines of evidence have verified an important role of MAPK in the pathogenesis of hepatic I/R injury (Sun et al. 2015, Nace et al. 2013), by regulating apoptosis and inflammation. However, the role of PKR on these pathways is still controversial. One study showed that TNF-induced JNK activation was abrogated, whereas activation of ERK and P38 was potentiated in PKR-deficient MEF cells (Takada et al. 2007). While other research revealed that PKR was essential for the activation of P38 in LPS-treated MEF (Goh et al. 2000) or TNF-treated chondrocytes (Ma et al. 2019). Our data determined that PKR was associated with enhanced I/R-induced activation of ERK and JNK but not p38 activation. Hence, the functions of PKR in the MAPK signaling pathway might be dependent on cell types and the extracellular stimuli as well as intracellular molecular events involved.

STAT family are intracellular messengers that relay different signals sensed the plasma membrane to specific genes in the nucleus, thus activating gene transcription (Sadowski et al. 1993). There are seven members of the STAT family, which include STAT1, STAT2, STAT3, STAT4, STAT5a, STAT5b, and STAT6 (Darnell 1997) and all contribute to innate and adaptive immunity (O'Shea et al. 2012). Interestingly, PKR has also been shown to participate in the regulation of various signal transduction pathways. However, several independent studies have presented opposing effects of PKR on STAT1 activity. PKR was shown to negatively regulate STAT1 in MEF cells stimulated with IFN-Y (Wang et al. 2006, Wong et al. 1997). While in other studies, STAT1 activation was observed to be dependent on PKR (Hsu et al. 2004, Ruuska et al. 2012). This controversy has not been completely resolved. In our study, we found that phosphorylated STAT1 protein levels were significantly lower with PKR deletion following I/R group demonstrating that PKR was required for the upregulation of STAT1 signaling in liver I/R insult. Furthermore, abolishing STAT1 with fludarabine also attenuated I/R-induced liver damage in comparison with the vehicle-treated I/R group. These findings are the first to link PKR and STAT1 in the acute setting of sterile injury induced by I/R. Additionally, fludarabine also has the potential to be used therapeutically to protect the liver from $\mathrm{I} / \mathrm{R}$ injury.

HMGB1 is a conserved protein that exists in the nucleus of almost all eukaryotic cells (Javaherian et al. 1978). We have detailed the role of extracellular HMGB1 as a driver of liver I/R-induced inflammation and injury when released by hypoxic hepatocytes (Tsung et al. 2005). However, intracellular HMGB1 upregulates protective autophagy in ischemic hepatocytes (Cardinal et al. 2009). Our present findings documented that PKR ablation or pharmacological inhibition of STAT1 reduced serum HMGB1 levels during hepatic I/R injury compared with their control groups. Taking together, these and previous observations establish that suppression of HMGB1 release may be crucial in protecting the liver against I/R injury. Although it remains unknown from our studies whether PKR induced HMGB1 release through active or passive mechanisms following I/R injury, our in vitro experiments established that PKR is 
involved in the active release of HMGB1 from hepatocytes exposed to hypoxia. We also provided evidence that pharmacological inhibition of JAK/STAT1 selectively prevented LPS-induced HMGB1 nuclear translocation and subsequent release (Lu et al. 2014). These findings support our previous findings that interventions that block HMGB1 activity could be effective in preventing l/R-induced liver damage (Tsung et al. 2005).

In summary, this study demonstrates that PKR is rapidly upregulated in the liver during ischemia and contributes to the STAT1 signaling upregulation and HMGB1 release. The association of all of the PKRrelated effects with increased liver damage points to PKR as a regulator of the intrinsic process that leads to secondary liver injury after I/R. Since inhibitors of PKR and STAT1 are available, these findings provide the rationale to consider these targets to reduce I/R-induced tissue injury.

\section{Abbreviations}

I/R, ischemia/reperfusion; PKR, double-stranded RNA dependent protein kinase (PKR); ALT, alanine aminotransferase; AST, aspartate aminotransferase; STAT, signal transducer and activator of transcription; HMGB1, high mobility group box-1; DAMPs, damage-associated molecular pattern molecules; PAMPs, pathogen-associated molecular pattern molecules; TNF-a, tumor necrosis factor-a; IL$1 \beta$, interleukin-1 $\beta$; MEFs, mouse embryonic fibroblasts; LPS, lipopolysaccharide; LDH, lactate dehydrogenase; IFN, Interferon; MAPK, mitogen-activated protein kinase; TUNEL, terminal deoxynucleotidyl transferase(TdT)-mediated dUTP nick-end labeling; JNK, c-Jun-N-terminal kinase; ERK, extracellular signal-regulated kinase; Bax, Bcl-2-associated X protein; Bcl2, B-cell lymphoma 2.

\section{Declarations}

\section{Ethics approval and consent to participate}

The current study was performed with the approval of the Ethics Committee of Central South University. All patients signed written informed consent before sample collection at the Second Xiangyang Hospital of Central South University.

\section{Consent for publication}

All authors read and agreed to the content of the final manuscript

\section{Availability of data and materials}

The datasets used and/or analyzed during the current study are available from the corresponding author on reasonable request.

\section{Competing interests}

The authors declare that they have no competing interests. 


\section{Funding}

This work was supported by National key scientific project 2015CB910700 (B.L.) and National Natural Science Foundation of China (No. 81930059 (B.L.).

\section{Author's Contributions}

B.L. and T.R.B. conceived the project and designed experiments and wrote the paper; D.T designed and performed the experiments, analyzed the data, and wrote the manuscript; Y.T. supervised the study; Y.J, X.C and X.Q performed the mouse experiments; D.L analyzed the data; W.L analyzed the data and put the figures together. W.C collected human liver samples.

\section{Acknowledgments}

Not applicable.

\section{References}

1. Zhang H, Chen T, Ren J, Xia Y, Onuma A, Wang Y, et al. Pre-operative exercise therapy triggers antiinflammatory trained immunity of Kupffer cells through metabolic reprogramming. Nature metabolism. 2021;3(6):843-58.

2. Zhang XJ, Cheng X, Yan ZZ, Fang J, Wang X, Wang W, et al. An ALOX12-12-HETE-GPR31 signaling axis is a key mediator of hepatic ischemia-reperfusion injury. Nature medicine. 2018;24(1):73-83.

3. Sun P, Zhang P, Wang PX, Zhu LH, Du Y, Tian S, et al. Mindin deficiency protects the liver against ischemia/reperfusion injury. Journal of hepatology. 2015;63(5):1198-211.

4. Liu Y, Lu T, Zhang C, Xu J, Xue Z, Busuttil RW, et al. Activation of YAP attenuates hepatic damage and fibrosis in liver ischemia-reperfusion injury. Journal of hepatology. 2019;71(4):719-30.

5. Yazdani HO, Kaltenmeier C, Morder K, Moon J, Traczek M, Loughran P, et al. Exercise Training Decreases Hepatic Injury and Metastases Through Changes in Immune Response to Liver Ischemia/Reperfusion in Mice. Hepatology (Baltimore, Md.). 2021;73(6):2494-509.

6. Jaeschke H, Woolbright BL. Current strategies to minimize hepatic ischemia-reperfusion injury by targeting reactive oxygen species. Transplantation reviews (Orlando, Fla.). 2012;26(2):103-14.

7. Meurs E, Chong K, Galabru J, Thomas NS, Kerr IM, Williams BR, et al. Molecular cloning and characterization of the human double-stranded RNA-activated protein kinase induced by interferon. Cell. 1990;62(2):379-90.

8. Holcik M, Sonenberg N. Translational control in stress and apoptosis. Nature reviews. Molecular cell biology. 2005;6(4):318-27.

9. Hsu LC, Park JM, Zhang K, Luo JL, Maeda S, Kaufman RJ, et al. The protein kinase PKR is required for macrophage apoptosis after activation of Toll-like receptor 4. Nature. 2004;428(6980):341-5. 
10. Yoon CH, Lee ES, Lim DS, Bae YS. PKR, a p53 target gene, plays a crucial role in the tumorsuppressor function of p53. Proceedings of the National Academy of Sciences of the United States of America. 2009;106(19):7852-7.

11. Kim SH, Gunnery S, Choe JK, Mathews MB. Neoplastic progression in melanoma and colon cancer is associated with increased expression and activity of the interferon-inducible protein kinase, PKR. Oncogene. 2002;21(57):8741-8.

12. Hugon J, Mouton-Liger F, Dumurgier J, Paquet C. PKR involvement in Alzheimer's disease. Alzheimer's research \& therapy. 2017;9(1):83.

13. Nakamura T, Furuhashi M, Li P, Cao H, Tuncman G, Sonenberg N, et al. Double-stranded RNAdependent protein kinase links pathogen sensing with stress and metabolic homeostasis. Cell. 2010;140(3):338-48.

14. Lu B, Nakamura T, Inouye K, Li J, Tang Y, Lundbäck P, et al. Novel role of PKR in inflammasome activation and HMGB1 release. Nature. 2012;488(7413):670-4.

15. Pan H, Ma Y, Wang D, Wang J, Jiang H, Pan S, et al. Effect of IFN-a on KC and LIX expression: role of STAT1 and its effect on neutrophil recruitment to the spleen after lipopolysaccharide stimulation. Molecular immunology. 2013;56(1-2):12-22.

16. Tsung A, Sahai R, Tanaka H, Nakao A, Fink MP, Lotze MT, et al. The nuclear factor HMGB1 mediates hepatic injury after murine liver ischemia-reperfusion. The Journal of experimental medicine. 2005;201(7):1135-43.

17. Li S, Yi Z, Deng M, Scott MJ, Yang C, Li W, et al. TSLP protects against liver I/R injury via activation of the PI3K/Akt pathway. JCl insight. 2019;4(22).

18. Suzuki S, Toledo-Pereyra LH, Rodriguez FJ, Cejalvo D. Neutrophil infiltration as an important factor in liver ischemia and reperfusion injury. Modulating effects of FK506 and cyclosporine. Transplantation. 1993;55(6):1265-72.

19. Yi Z, Deng M, Scott MJ, Fu G, Loughran PA, Lei Z, et al. IRG1/Itaconate Activates Nrf2 in Hepatocytes to Protect Against Liver Ischemia-Reperfusion Injury. Hepatology (Baltimore, Md.). 2020.

20. Nace GW, Huang H, Klune JR, Eid RE, Rosborough BR, Korff S, et al. Cellular-specific role of toll-like receptor 4 in hepatic ischemia-reperfusion injury in mice. Hepatology (Baltimore, Md.). 2013;58(1):374-87.

21. Brenner C, Galluzzi L, Kepp O, Kroemer G. Decoding cell death signals in liver inflammation. Journal of hepatology. 2013;59(3):583-94.

22. Wang S, Raven JF, Baltzis D, Kazemi S, Brunet DV, Hatzoglou M, et al. The catalytic activity of the eukaryotic initiation factor-2alpha kinase PKR is required to negatively regulate Stat1 and Stat3 via activation of the T-cell protein-tyrosine phosphatase. The Journal of biological chemistry. 2006;281(14):9439-49.

23. Lu B, Antoine DJ, Kwan K, Lundbäck P, Wähämaa $H$, Schierbeck $H$, et al. JAK/STAT1 signaling promotes HMGB1 hyperacetylation and nuclear translocation. Proceedings of the National Academy of Sciences of the United States of America. 2014;111(8):3068-73. 
24. Yeung MC, Liu J, Lau AS. An essential role for the interferon-inducible, double-stranded RNAactivated protein kinase PKR in the tumor necrosis factor-induced apoptosis in U937 cells. Proceedings of the National Academy of Sciences of the United States of America. 1996;93(22):12451-5.

25. Horng T, Barton GM, Medzhitov R. TIRAP: an adapter molecule in the Toll signaling pathway. Nature immunology. 2001;2(9):835-41.

26. Donzé 0 , Abbas-Terki T, Picard D. The Hsp90 chaperone complex is both a facilitator and a repressor of the dsRNA-dependent kinase PKR. The EMBO journal. 2001;20(14):3771-80.

27. Jammi NV, Whitby LR, Beal PA. Small molecule inhibitors of the RNA-dependent protein kinase. Biochemical and biophysical research communications. 2003;308(1):50-7.

28. Shimazawa M, Hara H. Inhibitor of double stranded RNA-dependent protein kinase protects against cell damage induced by ER stress. Neuroscience letters. 2006;409(3):192-5.

29. Xiao J, Tan Y, Li Y, Luo Y. The Specific Protein Kinase R (PKR) Inhibitor C16 Protects Neonatal Hypoxia-Ischemia Brain Damages by Inhibiting Neuroinflammation in a Neonatal Rat Model. Medical science monitor: international medical journal of experimental and clinical research. 2016;22(507481.

30. Watanabe T, Ninomiya H, Saitou T, Takanezawa S, Yamamoto S, Imai Y, et al. Therapeutic effects of the PKR inhibitor $\mathrm{C} 16$ suppressing tumor proliferation and angiogenesis in hepatocellular carcinoma in vitro and in vivo. Scientific reports. 2020;10(1):5133.

31. Couturier J, Paccalin M, Lafay-Chebassier C, Chalon S, Ingrand I, Pinguet J, et al. Pharmacological inhibition of PKR in APPswePS1dE9 mice transiently prevents inflammation at 12 months of age but increases $A \beta 42$ levels in the late stages of the Alzheimer's disease. Current Alzheimer research. 2012;9(3):344-60.

32. Li Y, Xiao J, Tan Y, Wang J, Zhang Y, Deng X, et al. Inhibition of PKR ameliorates lipopolysaccharideinduced acute lung injury by suppressing NF-KB pathway in mice. Immunopharmacology and immunotoxicology. 2017;39(4):165-72.

33. van Golen RF, Reiniers MJ, Olthof PB, van Gulik TM, Heger M. Sterile inflammation in hepatic ischemia/reperfusion injury: present concepts and potential therapeutics. Journal of gastroenterology and hepatology. 2013;28(3):394-400.

34. Hamilton G, Vogel S, Fuegger R, Gnant FX. Mechanisms of tumor necrosis factor-alpha and interleukin-6 induction during human liver transplantation. Mediators of inflammation. 1993;2(4):303-7.

35. Li G, Scull C, Ozcan L, Tabas I. NADPH oxidase links endoplasmic reticulum stress, oxidative stress, and PKR activation to induce apoptosis. The Journal of cell biology. 2010;191(6):1113-25.

36. Takada Y, Ichikawa H, Pataer A, Swisher S, Aggarwal BB. Genetic deletion of PKR abrogates TNFinduced activation of IkappaBalpha kinase, JNK, Akt and cell proliferation but potentiates p44/p42 MAPK and p38 MAPK activation. Oncogene. 2007;26(8):1201-12. 
37. Goh KC, deVeer MJ, Williams BR. The protein kinase PKR is required for p38 MAPK activation and the innate immune response to bacterial endotoxin. The EMBO journal. 2000;19(16):4292-7.

38. $\mathrm{Ma} \mathrm{CH}, \mathrm{Wu} \mathrm{CH}$, Jou IM, Tu YK, Hung CH, Chou WC, et al. PKR Promotes Oxidative Stress and Apoptosis of Human Articular Chondrocytes by Causing Mitochondrial Dysfunction through p38 MAPK Activation-PKR Activation Causes Apoptosis in Human Chondrocytes. Antioxidants (Basel, Switzerland). 2019;8(9).

39. Sadowski HB, Shuai K, Darnell JE, Jr., Gilman MZ. A common nuclear signal transduction pathway activated by growth factor and cytokine receptors. Science (New York, N.Y.). 1993;261(5129):173944.

40. Darnell JE, Jr. STATs and gene regulation. Science (New York, N.Y.). 1997;277(5332):1630-5.

41. O'Shea JJ, Plenge R. JAK and STAT signaling molecules in immunoregulation and immune-mediated disease. Immunity. 2012;36(4):542-50.

42. Wong AH, Tam NW, Yang YL, Cuddihy AR, Li S, Kirchhoff S, et al. Physical association between STAT1 and the interferon-inducible protein kinase PKR and implications for interferon and doublestranded RNA signaling pathways. The EMBO journal. 1997;16(6):1291-304.

43. Ruuska M, Sahlberg AS, Colbert RA, Granfors K, Penttinen MA. Enhanced phosphorylation of STAT-1 is dependent on double-stranded RNA-dependent protein kinase signaling in HLA-B27-expressing U937 monocytic cells. Arthritis and rheumatism. 2012;64(3):772-7.

44. Javaherian K, Liu JF, Wang JC. Nonhistone proteins HMG1 and HMG2 change the DNA helical structure. Science (New York, N.Y.). 1978;199(4335):1345-6.

45. Cardinal J, Pan P, Dhupar R, Ross $M$, Nakao A, Lotze $M$, et al. Cisplatin prevents high mobility group box 1 release and is protective in a murine model of hepatic ischemia/reperfusion injury. Hepatology (Baltimore, Md.). 2009;50(2):565-74.

\section{Figures}


A

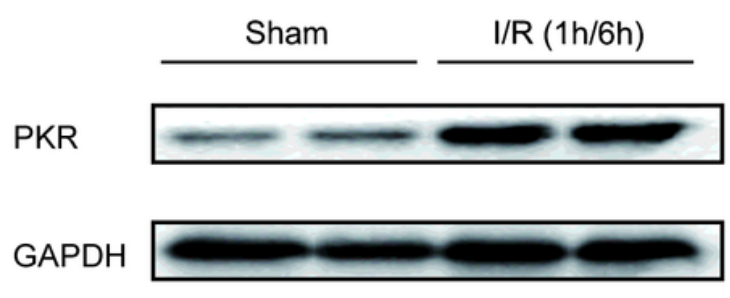

C

$$
\begin{array}{lllll}
\text { Case1 } & \text { Case2 } & \frac{\text { Case3 }}{\text { B I }} & \text { Case4 } \\
\hline
\end{array}
$$

PKR

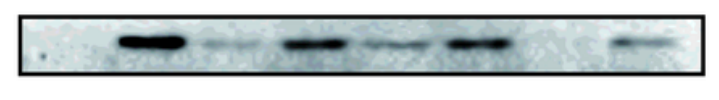

GAPDH

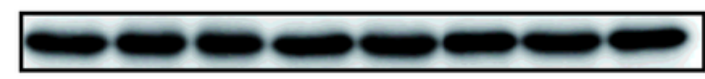

B:Baseline

I:Ischemia
B

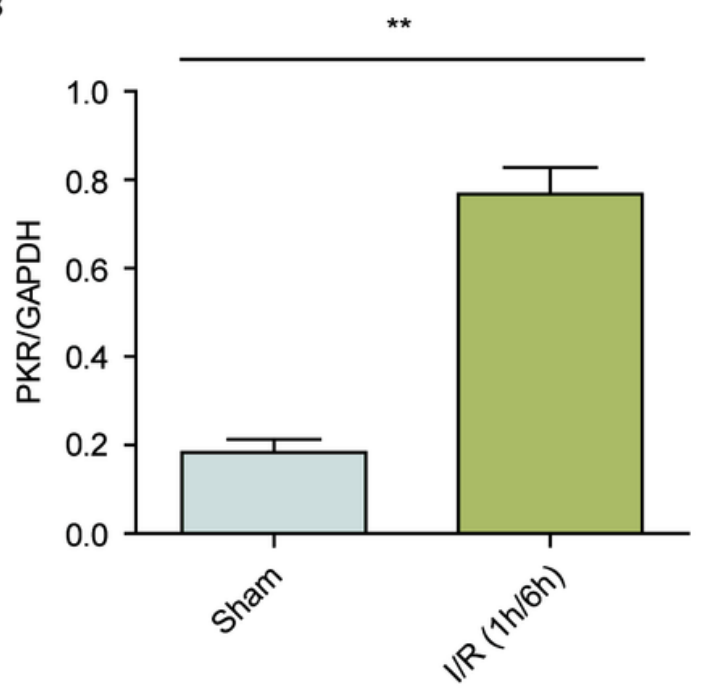

$\mathrm{D}$

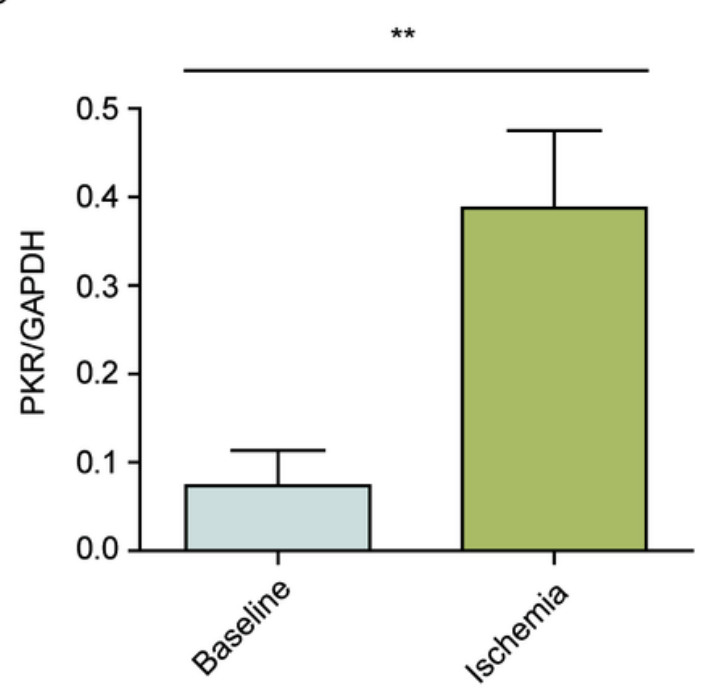

\section{Figure 1}

PKR is dramatically induced after liver I/R injury. (A, B) PKR protein expression levels in the livers of WT mice subjected to sham surgery or $1 \mathrm{~h}$ ischemia followed by $6 \mathrm{~h}$ reperfusion $(\mathrm{n}=6$ per group). (C, $D)$ Representative Western blot showing PKR protein expression in liver tissues of patients subjected to I/R during hepatic surgery. $B$, baseline (before ischemia); I, ischemia (after ischemia but before reperfusion); GAPDH served as a loading control. **P $₫ 0.01$. 

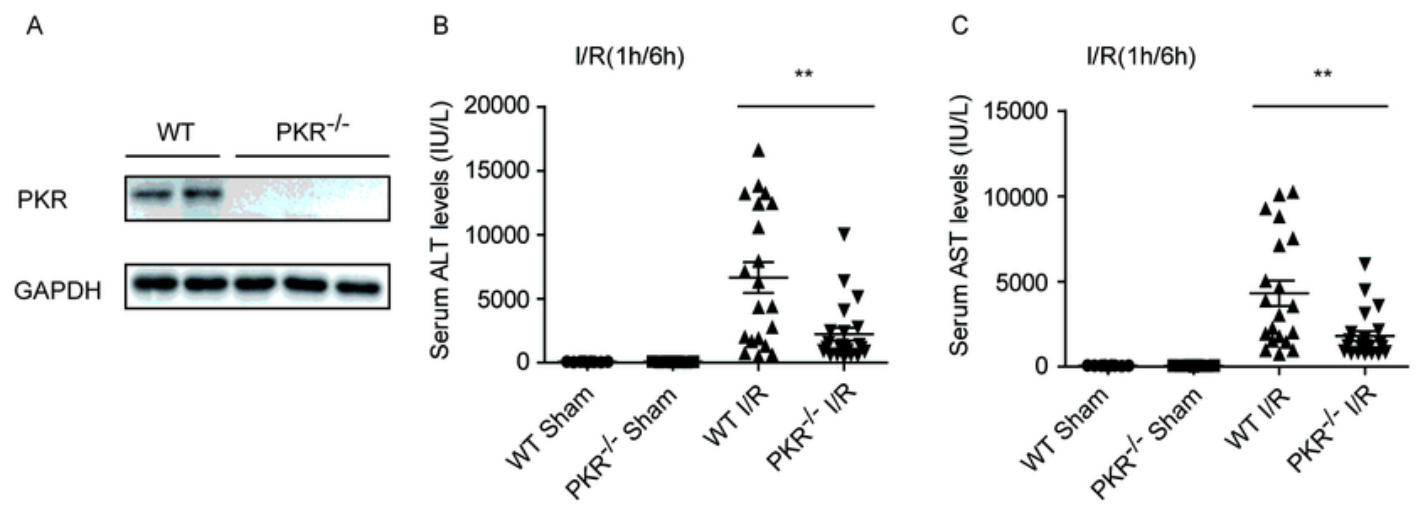

D
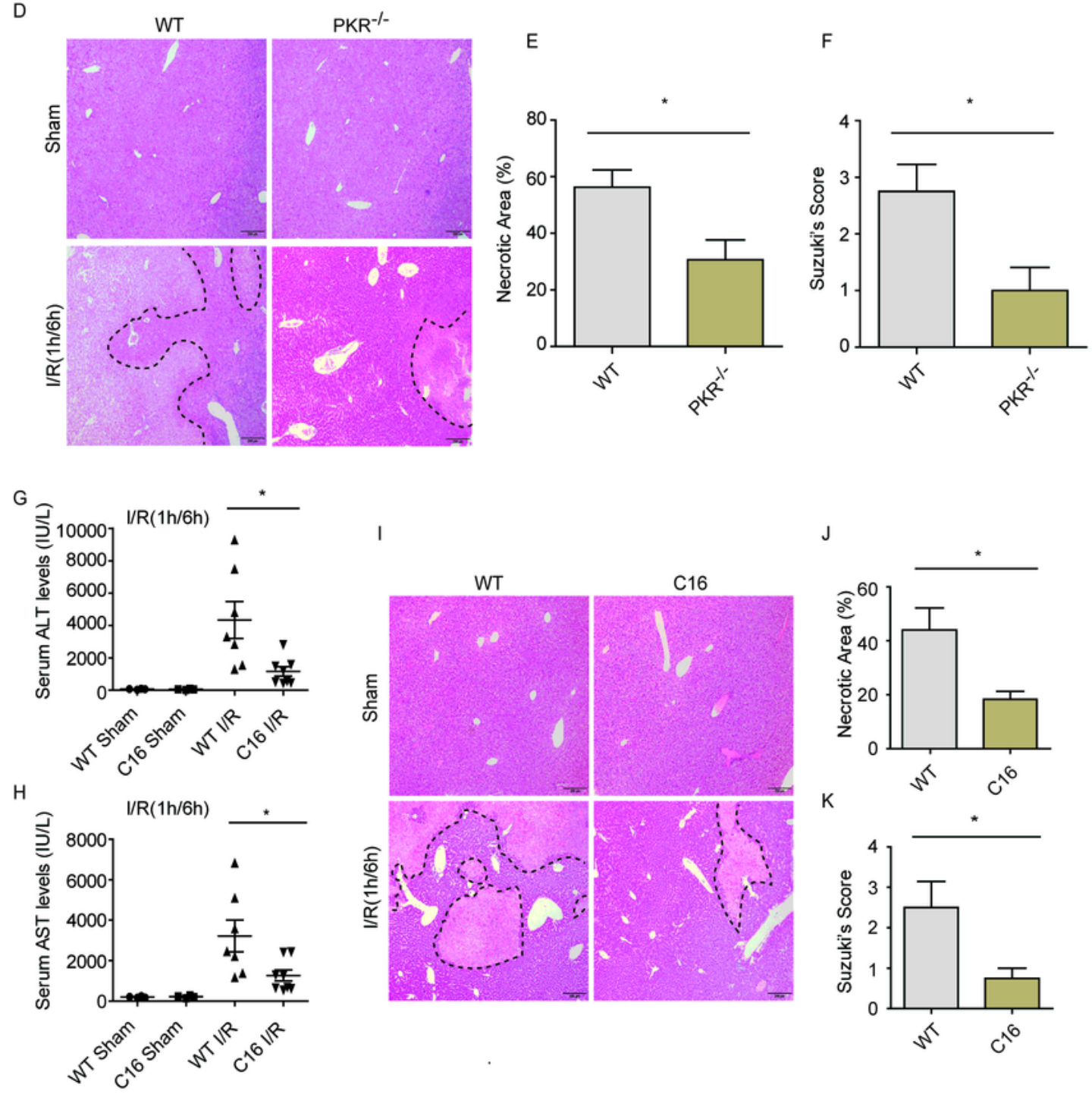

\section{Figure 2}

PKR deletion ameliorates hepatic I/R injury. (A) PKR protein levels in livers from WT and PKR-/- mice. (B, C) ALT(B) and AST(C) levels in the serum of WT and PKR-/- mice at $6 \mathrm{~h}$ after reperfusion or sham controls. $n=9$ for sham group, $n=20-22$ for I/R group. (D) Representative H\&E staining (10x) of liver lobes of WT and PKR-/- mice after sham operation or $6 \mathrm{~h}$ post-reperfusion. (E) Dotted lines in liver H\&E stained images indicated measured areas of necrosis, quantified in bar graph; $n=3-4$ for each group. ( $F$ ) Liver damages 
were assessed by Suzuki's histological score (I/R:1 h/6 h), quantified in bar graph; $\mathrm{n}=3-4$ for each group; $(G, H)$ Serum ALT $(G)$ and AST $(H)$ levels were measured in WT mice treated with vehicle or PKR inhibitor C16 and harvested $6 \mathrm{~h}$ after I/R; $\mathrm{n}=4$ for sham group, $\mathrm{n}=7-8$ for I/R group. (I) Representative H\&E staining of liver sections from vehicle-treated or C16-treated mice in the sham and I/R groups at $6 \mathrm{~h}$ postreperfusion ; $(J)$ Dotted lines in liver H\&E staining indicated necrotic areas, quantified in bar graph; $n=3-4$ for each group; $(K)$ Liver damages were analyzed by Suzuki's histological score (I/R:1 h/6 h), quantified in bar graph; $\mathrm{n}=3-4$ for each group. ${ }^{\star} \mathrm{P} \otimes 0.05,{ }^{\star *} \mathrm{P} \otimes 0.01$.

A

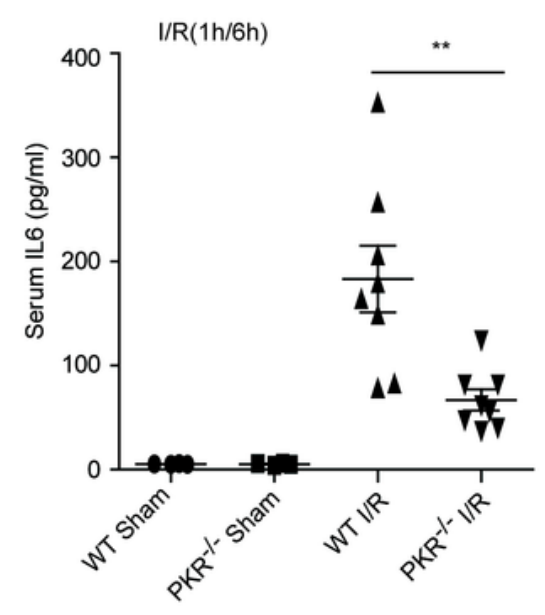

C

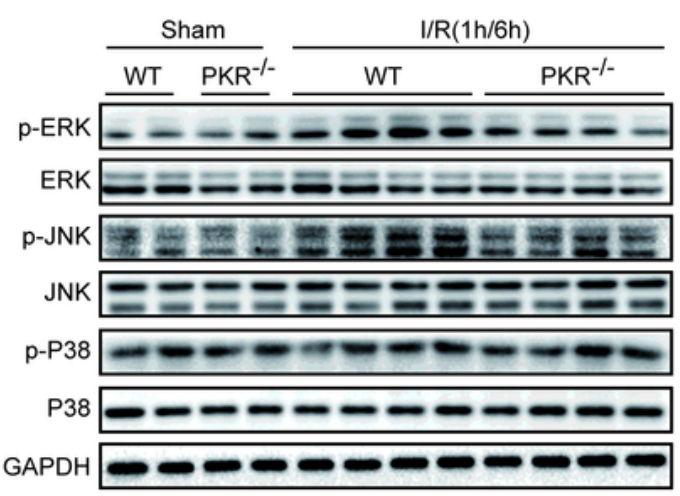

$E$

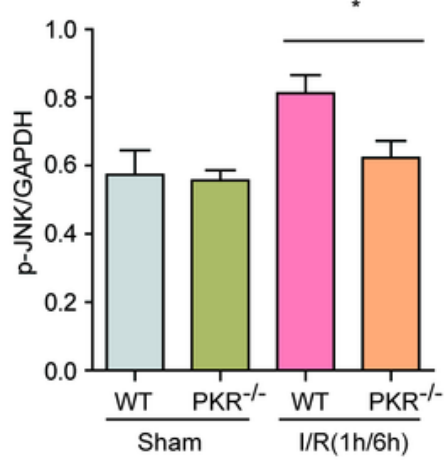

B

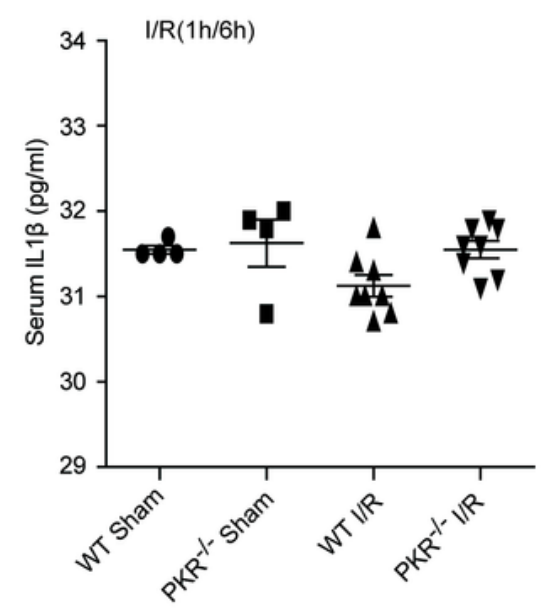

D

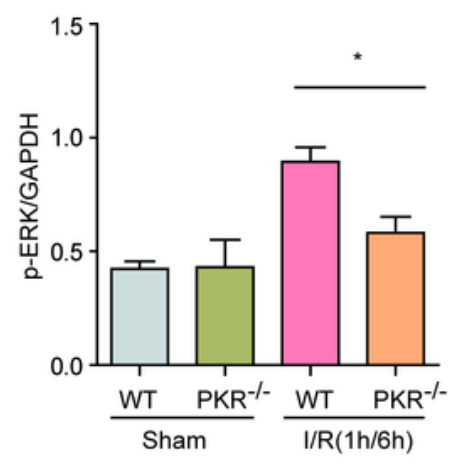

F

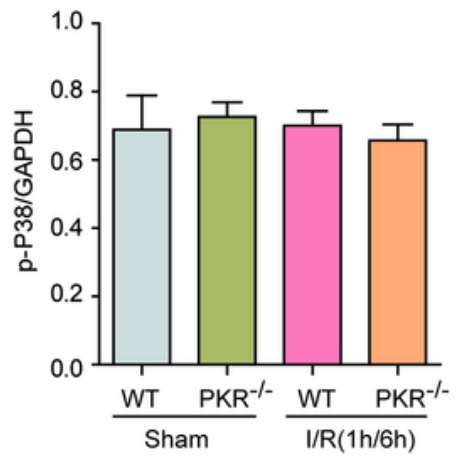




\section{Figure 3}

PKR increases hepatic I/R-induced inflammatory responses. (A, B) Serum levels of IL-6 (A) and IL-1 $\beta$ (B) in mice with PKR deficiency and the corresponding controls were assessed at $6 \mathrm{~h}$ post-reperfusion; $\mathrm{n}=4$ in each sham group, $n=8$ in each I/R group. (C) Western blotting analysis of the total and phosphorylated protein levels of ERK, JNK and p38 in mouse liver after I/R insult. (D-F) The protein expression levels of

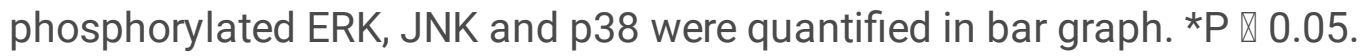

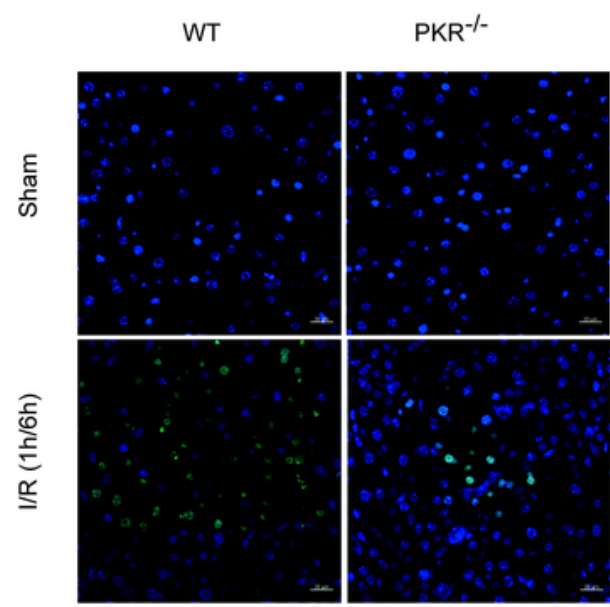

TUNEL(Green)/Nucleus(Blue)

C

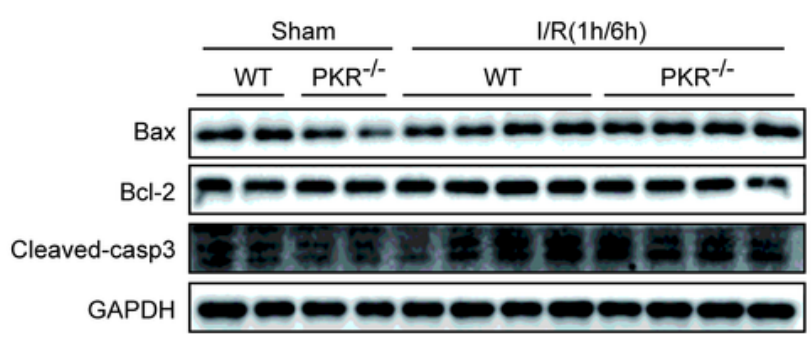

E

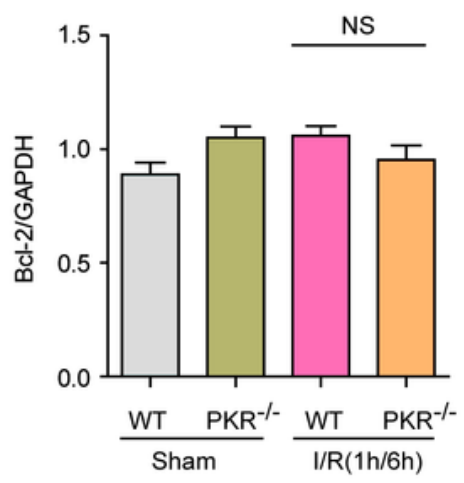

B

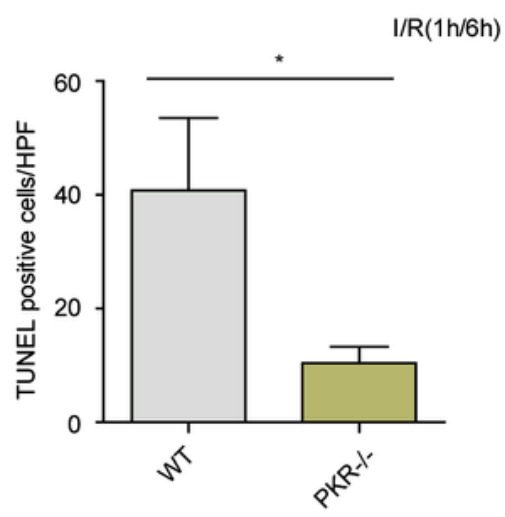

D
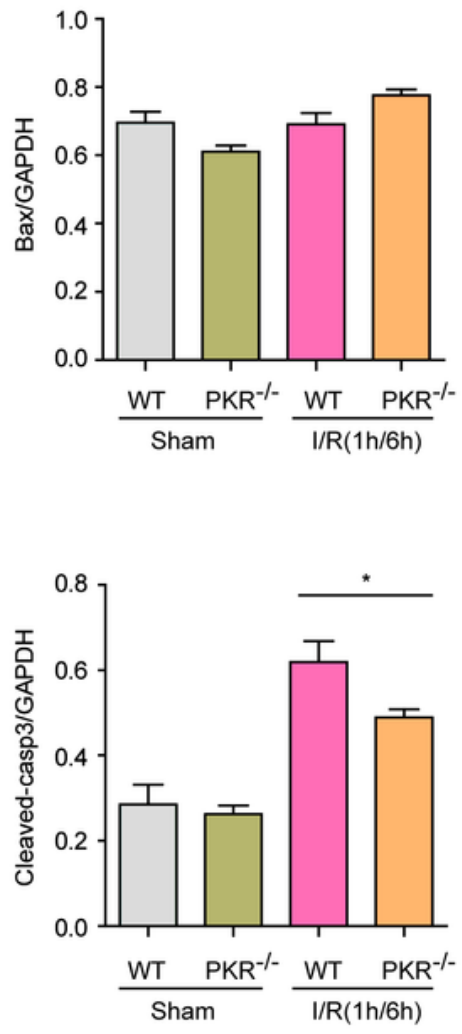
Figure 4

PKR promotes cell death in hepatic I/R injury. (A) TUNEL staining images (40x) of liver sections obtained from WT and PKR-/- mice at $6 \mathrm{~h}$ post-reperfusion. The numbers of TUNEL-positive cells were quantified in each high-power field $(n=3)$. (B) Bcl-2, Bax and cleaved caspase-3 in the liver samples from the indicated groups at $6 \mathrm{~h}$ after $\mathrm{l} / \mathrm{R}$ injury were analyzed by Western Blot. GAPDH served as a loading control. (D-F) The protein levels of $\mathrm{Bcl}-2, \mathrm{Bax}$ and cleaved caspase-3 were quantified in bar graph. ${ }^{\star} \mathrm{p}<0.05$

A

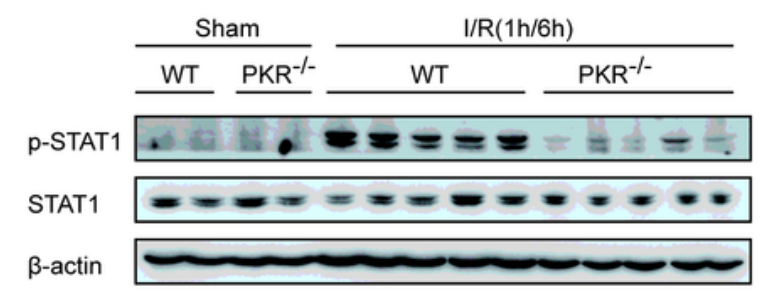

B

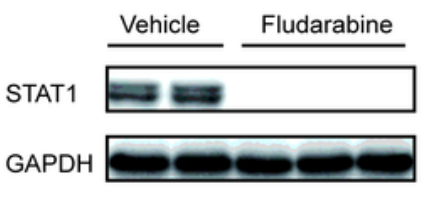

C

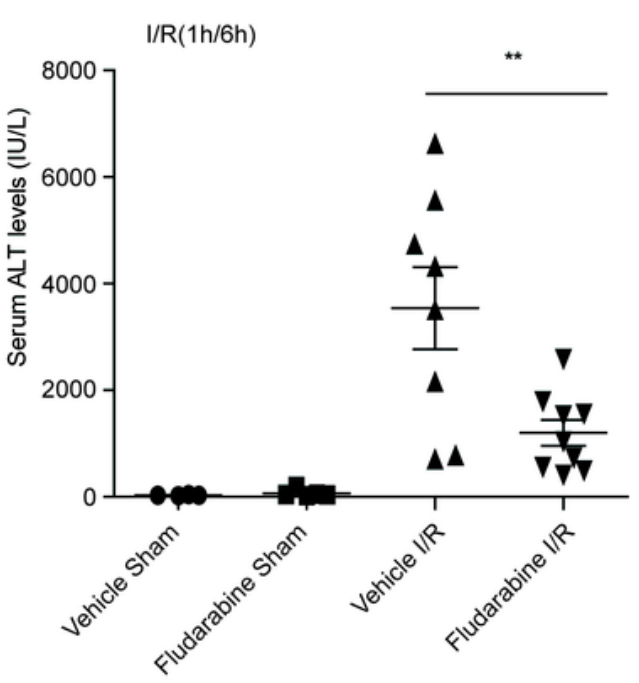

E

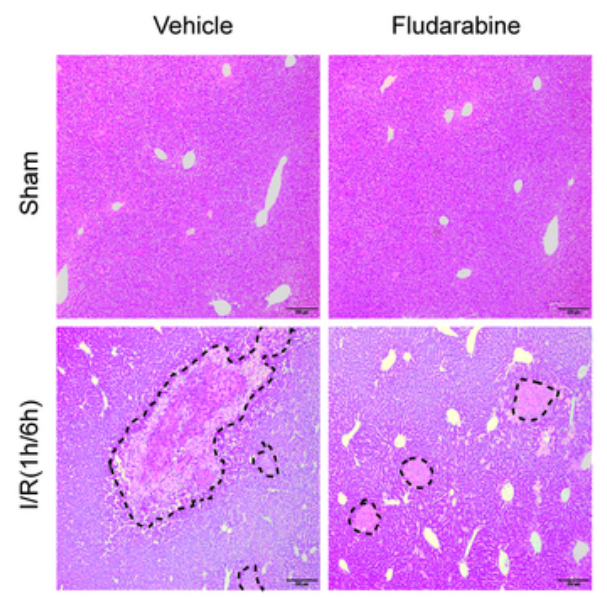

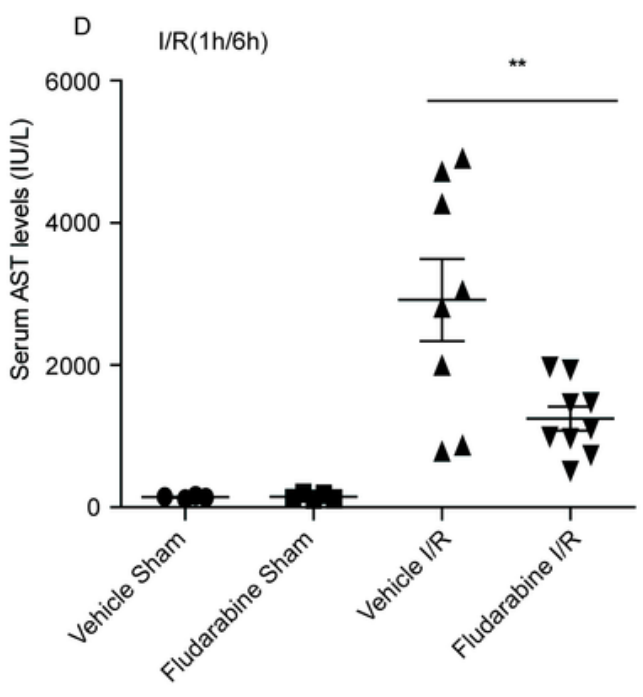

F

I/R(1h/6h)

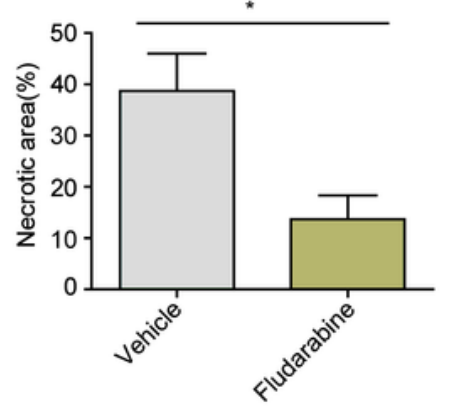

G

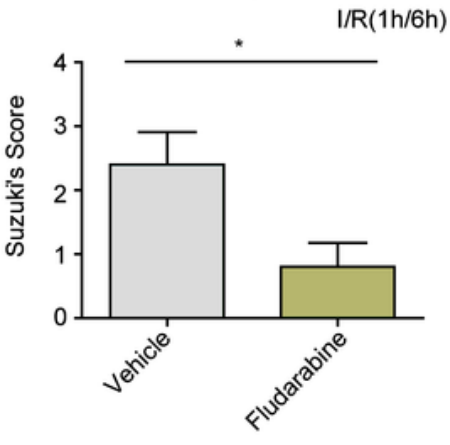




\section{Figure 5}

STAT1 inactivation blocks I/R-induced liver dysfunction of WT mice during hepatic I/R insult. (A) Total and phosphorylated STAT1 protein levels were evaluated in I/R-treated liver homogenates from WT and PKR-/- mice. (B) Western blot showing the levels of total STAT1 in WT mice subjected to the vehicle or fludarabine injection before I/R insult. (C, D) Serum ALT (C) and AST (D) levels were analyzed in WT mice treated with vehicle or fludarabine and harvested $6 \mathrm{~h}$ after I/R injury; $\mathrm{n}=4$ for the sham group, $\mathrm{n}=8-9$ for I/R group. (E-G) Representative H\&E staining liver sections (E), measured areas of necrosis (F) and Suzuki's histological scores $(G)$ in mice injected with vehicle and Fludarabine and subjected to I/R injury for $6 h . * P \otimes 0.05, * * P \otimes 0.01$. 
A

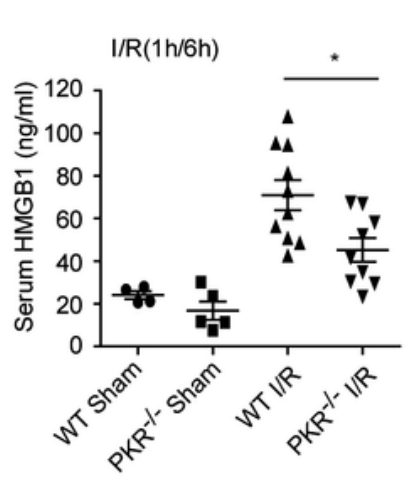

B

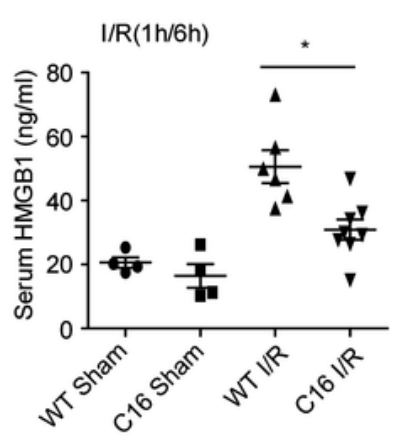

C

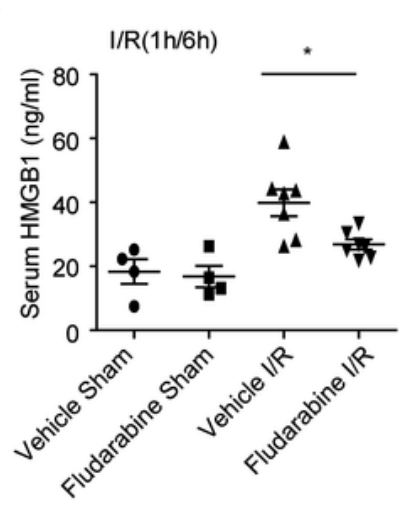

\section{Figure 6}

The release of HMGB1 is regulated by PKR. (A) HMGB1 in the serum of WT mice and PKR-/- mice after sham surgery or I/R injury analyzed by Elisa. (B) Serum HMGB1 levels in WT mice treated with vehicle or C16 and harvested 6h post-reperfusion evaluated by Elisa. (C) Serum HMGB1 levels in vehicle- or fludarabine-treated I/R group or sham operations were examined by Elisa. (D) HMGB1 visualized by immunofluorescent stain in WT and PKR-/- mouse hepatocytes subjected to normoxia or hypoxia. Red, 
HMGB1; Blue, nuclei; Green, F-actin. (E) Cultured hepatocytes were exposed to hypoxia (1\% O2) for $20 \mathrm{~h}$. Cell media was collected for Western blot analysis of HMGB1. (F) Cell death was assessed by LDH release from hepatocytes after normoxia or hypoxia. 